\title{
Analysis of biomarkers and metabolic pathways in patients with unstable angina based on ultra-high-performance liquid chromatography-quadrupole time-of-flight mass spectrometry
}

\author{
YUECHEN LIU ${ }^{1 *}$, YUE LI $^{2 *}$, TIANPU ZHANG ${ }^{1}$, HUAN ZHAO $^{1}$, SIMIAO FAN $^{1}$, XUEMENG CAI $^{2}$, \\ YIJIA LIU ${ }^{2}, \mathrm{ZHU} \mathrm{LI}^{2}, \mathrm{SHAN} \mathrm{GAO}^{2}$, YUBO LI ${ }^{1}$ and CHUNQUAN YU ${ }^{2}$ \\ ${ }^{1}$ College of Traditional Chinese Medicine, ${ }^{2}$ Research Institute of Traditional Chinese Medicine, \\ Tianjin University of Traditional Chinese Medicine, Tianjin 301617, P.R. China
}

Received September 19, 2019; Accepted June 26, 2020

DOI: $10.3892 / \mathrm{mmr} .2020 .11476$

\begin{abstract}
Unstable angina (UA) is a coronary disease with a high mortality and morbidity worldwide. The present study aimed to use non-invasive techniques to identify urine biomarkers in patients with UA, so as to provide more information for the early diagnosis and treatment of the disease. Based on metabolomics, urine samples from 28 patients with UA and 28 healthy controls (HCs) were analyzed using ultra-high-performance liquid chromatography-quadrupole time-of-flight mass spectrometry (UPLC-Q-TOF/MS). A total of 16 significant biomarkers that could distinguish between patients with UA and HCs, including D-glucuronic acid, creatinine, succinic acid and $\mathrm{N}$-acetylneuraminic acid, were identified. The major metabolic pathways associated with UA were subsequently analyzed by non-targeted metabolomics. The results demonstrated that amino acid and energy metabolism, fatty acid metabolism, purine metabolism and steroid hormone biosynthetic metabolism may serve important roles in UA. The results of the current study may provide a theoretical basis for the early diagnosis of UA and novel treatment strategies for clinicians. The trial was registered with the Chinese Clinical Trial Registration
\end{abstract}

Correspondence to: Professor Yubo Li, College of Traditional Chinese Medicine, Tianjin University of Traditional Chinese Medicine, 10 Poyanghu Road, Jinghai, Tianjin 301617, P.R. China E-mail: yaowufenxi001@sina.com

Professor Chunquan Yu, Research Institute of Traditional Chinese Medicine, Tianjin University of Traditional Chinese Medicine, 10 Poyanghu Road, Jinghai, Tianjin 301617, P.R. China

E-mail: ycqtjutcm@foxmail.com

*Contributed equally

Key words: unstable angina, urine, metabolomics, ultra-high-performance liquid chromatography-quadrupole time-of-flight mass spectrometry, biomarkers
Center (registration no. ChiCTR-ROC-17013957) at Tianjin University of Traditional Chinese Medicine.

\section{Introduction}

According to National Health and Nutrition Examination Survey data between 2011 and 2014, the prevalence of coronary heart disease (CHD) in the USA was $6.3 \%$ in adults $\geq 20$ years of age (1). Unstable angina (UA) is a severe and common complication of CHD. On the basis of administrative claims data for US Medicare beneficiaries, hospitalization for UA decreased from $1.5 \%$ in 1997 to $0.6 \%$ in 2009 (1). A previous study in Denmark has demonstrated that with the introduction of the high-sensitivity troponin test, the accuracy of UA diagnosis has been improved (2). Overall, $\sim 10 \%$ of all internal medicine emergency patients present to the emergency department with chest pain, but only $10 \%$ of these patients have an acute myocardial infarction as the underlying cause (3). UA is more severe compared with fatigue stable angina, but less severe compared with acute myocardial infarction (4). The main symptom of UA is severe chest pain, which can occur during rest (4). Coronary atherosclerotic plaque rupture, platelet aggregation and thrombosis are the main causes of coronary blood flow occlusion, which induces UA (5). Due to the unique pathophysiological mechanism UA, patients may develop acute myocardial ischemia or acute myocardial infarction if UA is not detected and treated in time (6). Therefore, the timely diagnosis of UA is essential. The diagnosis of UA is primarily based on electrocardiograms and the clinical symptoms of patients (7); however, the subjective nature of clinical symptoms and transient changes of electrocardiograms are limitations. Although coronary angiography is a reliable and accurate diagnostic method for UA, it is an invasive procedure that may be unacceptable for certain patients. Therefore, there is an urgent requirement for a non-invasive technique to diagnose UA quickly and accurately.

As an emerging method of systematic biological technology, metabolomics has provided application prospects in a range of subject areas, such as disease pathophysiological process research and molecular diagnosis of cancer $(8,9)$. Elucidating differences between healthy and diseased states 
is of important clinical use (10). Metabolomics techniques aim to quantitatively measure the systemic effects of endogenous small molecule metabolites produced by exogenous substances in the body $(11,12)$. Additionally, it elucidates pathological processes and the dynamic metabolic responses of substances in the body (13). The use of metabolomics to identify potential disease biomarkers has allowed for early diagnoses and the determination of associated biological mechanisms $(14,15)$. In a recent study, the phospholipid profile in serum of patients with Alzheimer's disease was studied by multi-platform metabonomics to find a new diagnostic method (16). In this study, sera from 19 patients with Alzheimer's disease and 17 healthy volunteers were collected and significant changes were detected in the levels of several phosphatidylcholine, phosphatidylethanolamine, plasma alkenylcholine, plasma alkenylethanolamine and different lysophospholipids, which provided a global insight into the possible factors that triggered membrane rupture, a possible underlying mechanism of Alzheimer's disease. Although the human urine metabolome is a subset of the human serum metabolome, compounds that are below the limit of detection in the blood are detectable in urine $(17,18)$. Urine is therefore becoming a surrogate to blood for monitoring disease biomarkers. Samples are easy to collect, repeatedly available and can be obtained in large quantities from patients of all ages. Urine also contains low cell and protein content, and rich chemical compositions, which provides valuable bioinformation on metabolism $(19,20)$. In recent years, metabolomics has made progress in cardiovascular research. Wang et al (21) used ultra-high-performance liquid chromatography-quadrupole time-of-flight mass spectrometry (UPLC-Q-TOF/MS) to conduct urine metabolomics on patients with acute coronary syndrome. The results revealed that nine biomarkers, such as isobutyryl-1-carnitine, were significantly upregulated, and that the expression of 11 biomarkers, including 1-lactic acid, were downregulated; in addition, fatty acid metabolism, fatty acid $\beta$-oxidation metabolism, amino acid metabolism and the tricarboxylic acid (TCA) cycle were demonstrated to serve important roles in acute coronary syndrome. Li et al (22) also performed UPLC-Q-TOF/MS for metabolomics, and the results revealed the interacting mechanism between Danhong injection and low-dose aspirin, providing a reference for cardiovascular disease research, which suggested that the emerging alterations caused by interaction between Danhong injection and low-dose aspirin might influence the co-administration of other drugs or the treatment of relevant diseases. Urine metabolomics has therefore become a powerful non-invasive technique for diagnosing and monitoring various human diseases $(18,23)$.

The current study aimed to use a UPLC-Q-TOF/MS-based untargeted metabolomics platform to screen urine biomarkers in patients with UA by principal component analysis (PCA) and partial least squares discriminant analysis (PLS-DA). Receiver operating characteristic (ROC) curves were used to determine whether these biomarkers exhibited diagnostic significance for UA, and changes in metabolic pathways during UA were examined using the metabolomics pathway analysis (MetPA) web-based tool. The present findings provide new insights into UA pathogenesis and its clinical diagnosis.

\section{Materials and methods}

Clinical trial registration and ethics statement. A total of 28 patients with UA and 28 healthy controls (HCs) were recruited from Tianjin Chest Hospital (Tianjin, China) and the General Hospital of Tianjin Medical University (Tianjin, China). All participants enrolled in the current study provided written informed consent. The present study conformed to the Declaration of Helsinki and was approved by the Ethics Committee of Tianjin University of Traditional Chinese Medicine (approval no. TJUTCM-EC20180003).

Inclusion criteria. According to the Guidelines for the Diagnosis and Treatment of Unstable Angina and Non-ST-Segment Elevation Myocardial Infarction issued by the Chinese Medical Association Cardiovascular Branch and the Editorial Board of the Chinese Journal of Cardiovascular Diseases in 2007 (24), all patients were enrolled with the following subtypes: i) Resting angina (the episode occurs at rest with a duration $>20 \mathrm{~min}$; ii) initial angina (new angina occurring within 1 month); iii) worsening exertional angina pectoris (a history of angina pectoris with pain worsening in the last month); and iv) variant angina pectoris (transient ST-segment elevation with self-remission). The age-matched HCs adhered to strict standards for inclusion: i) No abnormalities detected during physical examination, including blood pressure, height, weight, gait and appearance; ii) no abnormal medical history, family history or female reproductive history; and iii) no abnormalities in any of the following examinations: Cardiopulmonary, physical, electrocardiogram, blood (routine, blood glucose, blood lipid, liver function and renal function) and special examination for female gynecology.

Exclusion criteria for patients. All patients were aged between 45-65 years and met the diagnostic criteria for UA. Patients were excluded if they: i) Had diseases that may cause chest pain, such as other heart diseases, psychoneuroses, menopause, hyperthyroidism, cervical spondylotic myelopathy, vertebral arteriopathy cervical spondylosis, gastro-esophageal reflux disease or hiatal hernia; ii) used $>3$ of the four types of drugs: $\beta$-receptor blockers, calcium channel antagonists, energy metabolism drugs and nitrate drugs; iii) exhibited hypertension following antihypertensive drug treatment [systolic blood pressure $(\mathrm{SBP}) \geq 160 \mathrm{mmHg}$, diastolic blood pressure (DBP) $\geq 100 \mathrm{mmHg}$ ], severe cardiopulmonary insufficiency or severe arrhythmia (rapid atrial fibrillation, atrial flutter and paroxysmal ventricular tachycardia); iv) presented active liver disease or unexplained elevated serum transaminase and alanine aminotransferase levels, with aspartate aminotransferase twice the upper limit of the normal reference value; v) had renal dysfunction; vi) exhibited severe primary disease such as hematopoietic system tumors or malignant tumors; vii) were pregnant, lactating or of childbearing age and preparing for pregnancy; viii) were mentally ill or exhibited impaired cognition; ix) had severe metabolic diseases such as diabetic or gouty nephropathy; and $\mathrm{x}$ ) participated in other clinical trials within the last 3 months.

Processing and preparation of sample. Fasting morning blood was collected in the Tianjin Chest Hospital and the General 
Hospital of Tianjin Medical University and stored at $4^{\circ} \mathrm{C}$. Fasting morning urine was collected from the subjects and centrifuged at $4^{\circ} \mathrm{C}$ and $1,370 \mathrm{x}$ g for 15 min within $2 \mathrm{~h}$. The supernatant was removed and centrifuged at $1,000 \mathrm{x}$ g for $8 \mathrm{~min}$ at $4{ }^{\circ} \mathrm{C}$. Subsequently, sodium azide was added to the sample at a volume ratio of $1: 100$ for preservation at $-80^{\circ} \mathrm{C}$ for the metabonomic analysis. Each group of samples was completely thawed at room temperature, and $300 \mu 1$ of each sample was centrifuged at $4^{\circ} \mathrm{C}$ and $11,180 \mathrm{x}$ g for $10 \mathrm{~min}$. Subsequently, $150 \mu \mathrm{l}$ of the supernatant was added and to $150 \mu 1$ distilled water and vortexed for $1 \mathrm{~min}$. Following centrifugation at $18,890 \mathrm{x}$ g for $15 \mathrm{~min}, 200 \mu \mathrm{l}$ of the supernatant was analyzed in the vial for metabolomic analysis. In addition, $100 \mu 1$ of each urine sample was pipetted into a centrifuge tube to prepare quality control (QC) samples to validate the experimental precision, repeatability and stability.

UPLC- $Q-T O F / M S$. Data were acquired using a UPLC-Q-TOF/MS system (Waters Corporation). UPLC analysis was performed in a Waters Acquity UPLC system (Waters Corporation). MS was performed on a Waters Micro mass Q/TOF micro Synapt High Definition Mass Spectrometer. Detailed parameters of the instrument are presented in the Supplementary Materials and Methods (Data S1).

Statistical analysis. Raw UPLC-Q-TOF/MS data were subjected to peak discovery, peak alignment and peak filtering through the MarkerLynx applications manager data processing system (version 4.1; Waters Corporation) for the identification of potential discriminant variables. Subsequently, processed data (Excel format) were imported into SIMCA-P 12.0 statistical software (Umetrics; Sartorius AG), and unsupervised PCA was performed using SIMCA-P software. If PCA could not completely separate data, a supervised orthogonal PLS-DA was performed.

$\mathrm{M} / \mathrm{z}$ values of the candidate markers were searched in the human metabolome database (HMDB; http://www.hmdb. $\mathrm{ca} /$ ) and were further determined based on the secondary fragments obtained from the mass spectrum. The 'Statistical Analysis' (MetaboAnalyst 4.0; https://www.metaboanalyst.ca/) was used to analyze the correlation between the biomarkers and Pearson correlation analysis was used, and a correlation coefficient (Pearson's $r$ ) $>0.3$ was considered to indicate a correlation if $\mathrm{P}<0.05$. The 'Pathway Analysis' online tool (MetaboAnalyst 4.0) was used to identify the metabolic pathways associated with potential biomarkers.

ROC curves (IBM SPSS Statistics 21.0, IBM Corp.) were used to determine whether these biomarkers exhibited diagnostic significance for UA. The ROC curve had sensitivity (true-positive rate) as the ordinate and 1-specificity (false-positive rate) as the abscissa, which allows for the visual observation of the association between sensitivity and specificity. The area under the curve (AUC), reflects the diagnostic value of the biomarker. The closer the AUC value is to 1, the more effective the diagnostic effect.

Data between the UA and HC groups were compared by independent sample Student's t-test using SPSS 21.0. Data are presented as the mean \pm SD for Gaussian variables or as the median and IQR when normality was not tenable. $\mathrm{P}<0.05$ was considered to indicate a statistically significant difference.
Table I. Demographic information of patients with UA and HCs.

\begin{tabular}{lccc}
\hline Parameter & UA $(\mathrm{n}=28)$ & $\mathrm{HC}(\mathrm{n}=28)$ & P-value \\
\hline Age, years & $51.86 \pm 3.83$ & $50.18 \pm 5.29$ & 0.179 \\
Sex, male/female & $19 / 9$ & $15 / 13$ & 0.412 \\
Height, cm & $169.43 \pm 7.14$ & $165.93 \pm 7.72$ & 0.083 \\
SBP, $\mathrm{mmHg}$ & $132.32 \pm 11.85$ & $122.68 \pm 9.53$ & 0.001 \\
DBP, mmHg & $81.96 \pm 11.01$ & $77.14 \pm 5.77$ & 0.045 \\
\hline
\end{tabular}

Data are presented as the mean $\pm \mathrm{SD}$. UA, unstable angina; $\mathrm{HC}$, healthy control; SBP, systolic blood pressure; DBP, diastolic blood pressure.

Table II. Quality control experiment results.

\begin{tabular}{lcc}
\hline Experiment & Peak area, \% & Retention time, \% \\
\hline Instrument precision & 0.10 & 0.89 \\
Method repeatability & 0.10 & 0.98 \\
Sample stability & 1.88 & 1.47 \\
\hline
\end{tabular}

\section{Results}

Demographic information. No significant differences were observed between patients with UA and the HCs in age or height $(\mathrm{P}>0.05)$. SBP and DBP levels were higher in the UA group compared with those in the HCs $(\mathrm{P}<0.05)$. The detailed demographic analysis results are presented in Table I. The biochemical indicators of the subjects in the HC group are listed in supplementary materials (Table SI), all values of blood glucose, blood lipid and renal function were within the normal range. The medications administered to patients with UA following blood collection are presented in Table SII, including antiplatelet drugs, statins drugs, beta blocker drugs, nitrates drugs, energy metabolism drugs, and so on.

Metabolomics analysis. The relative standard deviation (RSD) of peak areas and retention times of 20 randomly selected chromatographic peaks were $<15 \%$, indicating that metabolomics requirements were met. The $\mathrm{QC}$ results are presented in Table II. Based on UPLC-Q-TOF/MS analysis technology, the base peak intensity (BPI) chromatograms of urine metabolomics were obtained from 56 patients with UA and HCs, as well as QC samples. The positive and negative ion maps of the QC samples are presented in Fig. 1, and the PCA score map is presented in Fig. 2A and C. It can be observed from the PLS-DA score plots in Fig. 2B and D that the samples from different groups were sorted into different quadrants, with no overlap, which demonstrated that a wellfitted PLS-DA model was established. The two groups of samples exhibited acceptable classification and aggregation, indicating that the metabolic patterns of the UA and $\mathrm{HC}$ groups were different. 
Table III. Metabolites associated with unstable angina based on ultra-high-performance liquid chromatography-quadrupole time-of-flight mass spectrometry.

\begin{tabular}{|c|c|c|c|c|c|c|c|}
\hline Marker no. & $\mathrm{RT}, \min$ & Observed, m/z & Calculated, $\mathrm{m} / \mathrm{z}$ & Compound & Molecular formula & Parent ion & Change \\
\hline 1 & 1.8841 & 330.0704 & 330.0736 & Glutathione & $\mathrm{C}_{10} \mathrm{H}_{17} \mathrm{O}_{6} \mathrm{~N}_{3} \mathrm{~S}$ & $\mathrm{M}+\mathrm{Na}$ & $\downarrow^{\mathrm{b}}$ \\
\hline 2 & 0.8751 & 117.0177 & 117.0188 & Succinic acid & $\mathrm{C}_{4} \mathrm{H}_{6} \mathrm{O}_{4}$ & M-H & $\uparrow^{b}$ \\
\hline 3 & 1.1444 & 103.0393 & 103.0395 & Succinic acid semialdehyde & $\mathrm{C}_{4} \mathrm{H}_{6} \mathrm{O}_{3}$ & $\mathrm{M}+\mathrm{H}$ & $\downarrow^{\mathrm{b}}$ \\
\hline 4 & 4.2378 & 112.0505 & 112.0511 & Creatinine & $\mathrm{C}_{4} \mathrm{H}_{7} \mathrm{~N}_{3} \mathrm{O}$ & $\mathrm{M}-\mathrm{H}$ & $\uparrow^{\mathrm{a}}$ \\
\hline 5 & 7.3885 & 175.0239 & 175.0243 & D-Glucurono-6,3-lactone & $\mathrm{C}_{6} \mathrm{H}_{8} \mathrm{O}_{6}$ & $\mathrm{M}-\mathrm{H}$ & $\downarrow^{\mathrm{a}}$ \\
\hline 6 & 4.2493 & 193.0348 & 193.0348 & D-Glucuronic acid & $\mathrm{C}_{6} \mathrm{H}_{10} \mathrm{O}_{7}$ & $\mathrm{M}-\mathrm{H}$ & $\uparrow^{\mathrm{a}}$ \\
\hline 7 & 0.8662 & 308.0953 & 308.0982 & N-Acetylneuraminic acid & $\mathrm{C}_{11} \mathrm{H}_{19} \mathrm{NO}_{9}$ & $\mathrm{M}-\mathrm{H}$ & $\uparrow^{b}$ \\
\hline 8 & 5.7765 & 171.0642 & 171.0657 & 2-Octenedioic acid & $\mathrm{C}_{8} \mathrm{H}_{12} \mathrm{O}_{4}$ & $\mathrm{M}-\mathrm{H}$ & $\uparrow^{\mathrm{a}}$ \\
\hline 9 & 11.2255 & 327.2304 & 327.2300 & Arachidonic acid & $\mathrm{C}_{20} \mathrm{H}_{32} \mathrm{O}_{2}$ & $\mathrm{M}+\mathrm{Na}$ & $\uparrow^{b}$ \\
\hline 10 & 0.8245 & 131.0345 & 131.0344 & Glutaric acid & $\mathrm{C}_{5} \mathrm{H}_{8} \mathrm{O}_{4}$ & $\mathrm{M}-\mathrm{H}$ & $\uparrow^{b}$ \\
\hline 11 & 10.6419 & 201.1108 & 201.1127 & Sebacic acid & $\mathrm{C}_{10} \mathrm{H}_{18} \mathrm{O}_{4}$ & M-H & $\uparrow^{\mathrm{a}}$ \\
\hline 12 & 11.3095 & 273.1864 & 273.1855 & Estradiol & $\mathrm{C}_{18} \mathrm{H}_{24} \mathrm{O}_{2}$ & $\mathrm{M}+\mathrm{H}$ & $\uparrow^{b}$ \\
\hline 13 & 2.8365 & 225.0855 & 225.0875 & Porphobilinogen & $\mathrm{C}_{10} \mathrm{H}_{14} \mathrm{~N}_{2} \mathrm{O}_{4}$ & $\mathrm{M}-\mathrm{H}$ & $\downarrow^{\mathrm{b}}$ \\
\hline 14 & 11.5345 & 313.2173 & 313.2143 & Dihydrotestosterone & $\mathrm{C}_{19} \mathrm{H}_{30} \mathrm{O}_{2}$ & $\mathrm{M}+\mathrm{Na}$ & $\downarrow^{\mathrm{b}}$ \\
\hline 15 & 9.7406 & 363.2197 & 363.2171 & Cortisol & $\mathrm{C}_{21} \mathrm{H}_{30} \mathrm{O}_{5}$ & $\mathrm{M}+\mathrm{H}$ & $\downarrow^{\mathrm{b}}$ \\
\hline 16 & 3.3935 & 134.0480 & 134.0467 & Adenine & $\mathrm{C}_{5} \mathrm{H}_{5} \mathrm{~N}_{5}$ & $\mathrm{M}-\mathrm{H}$ & $\uparrow^{\mathrm{a}}$ \\
\hline
\end{tabular}

${ }^{\mathrm{a}} \mathrm{P}<0.05$ and ${ }^{\mathrm{b}} \mathrm{P}<0.01$ vs. healthy controls. $\uparrow$, content increased significantly; $\downarrow$, content decreased significantly. RT, retention time.
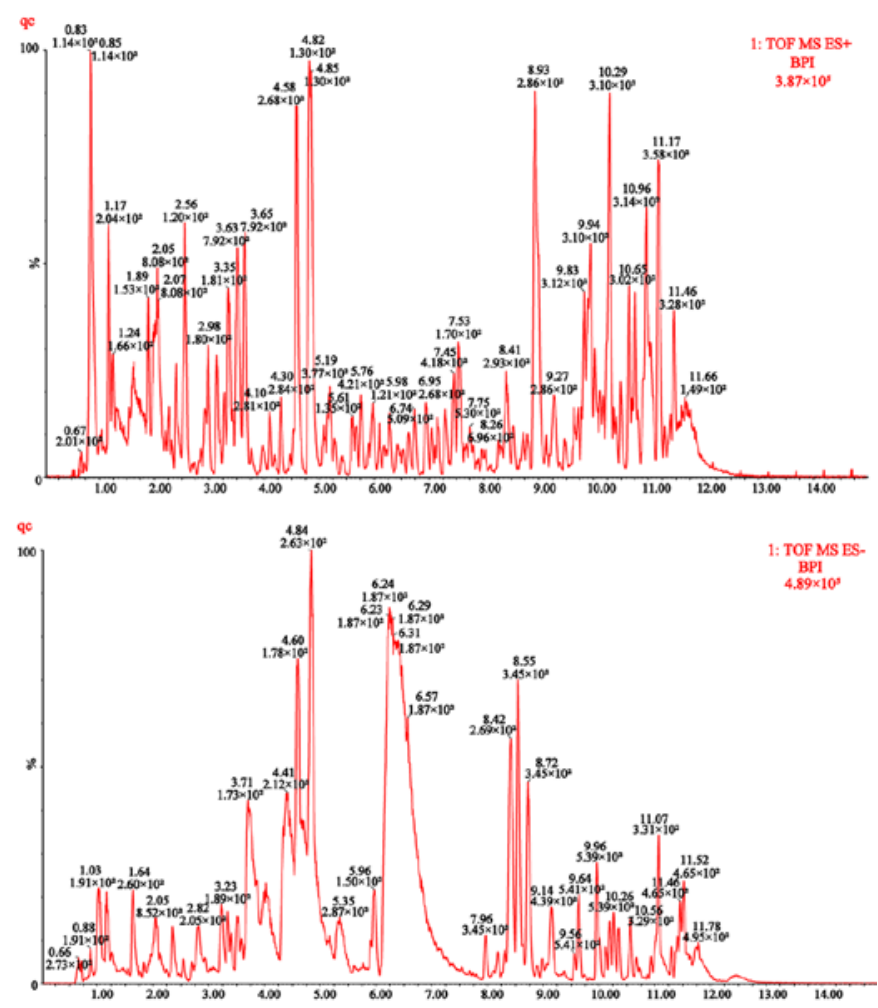

Figure 1. BPI chromatogram of urine in the QC samples with positive (top) and negative ion mode (bottom) based on ultra-high-performance liquid chromatography-quadrupole TOF/MS. qc, quality control; TOF, time-of-flight; MS, mass spectrometry; BPI, base peak intensity; ES, electrospray.

Identification of potential biomarkers. According to the PLS-DA model and the t-test results, substances with significant differences between the UA and HC groups were screened. Subsequently, differentially expressed small molecules were identified in patients with UA based on the $\mathrm{m} / \mathrm{z}$ values obtained from the HMDB. The urine biomarkers of 16 patients with UA were analyzed by comparing the secondary characteristic fragments of compounds such as D-glucuronic acid, creatinine, succinic acid and $\mathrm{N}$-acetylneuraminic acid; the details are presented in Table III. Compared with the HC groups, the levels of biomarkers such as glutathione, succinic acid semialdehyde, dihydrotestosterone and cortisol in the UA group decreased significantly, whereas the levels of succinic acid, $\mathrm{N}$-acetylneuraminic acid, arachidonic acid, glutaric acid and estradiol increased significantly. The metabolic pathways can be further analyzed through the change trend of the biomarkers, providing insight into the biological relevance of these markers for UA.

Analysis of potential biomarker metabolic pathways. Small molecule metabolites were screened in patients with UA to further elucidate the underlying metabolic pathways of the disease. The association and metabolic pathways between the small molecule metabolites were clarified using MetPA (Fig. 3). Metabolic pathways with a greater contribution to the effects of UA relative to the $\mathrm{HC}$ groups were considered to be involved in UA pathogenesis. The following pathways were identified: 'Alanine, aspartate and glutamate metabolism'; 'steroid hormone biosynthesis'; 'butanoate metabolism', 'ascorbate and aldarate metabolism'; 'tyrosine metabolism'; 'the citrate cycle'; 'glutathione metabolism'; 'lysine degradation', 'pentose and glucuronate interconversions'; and 'arachidonic acid metabolism'. These may be the main pathways that lead to the metabolic disorders of UA pectoris. 
A

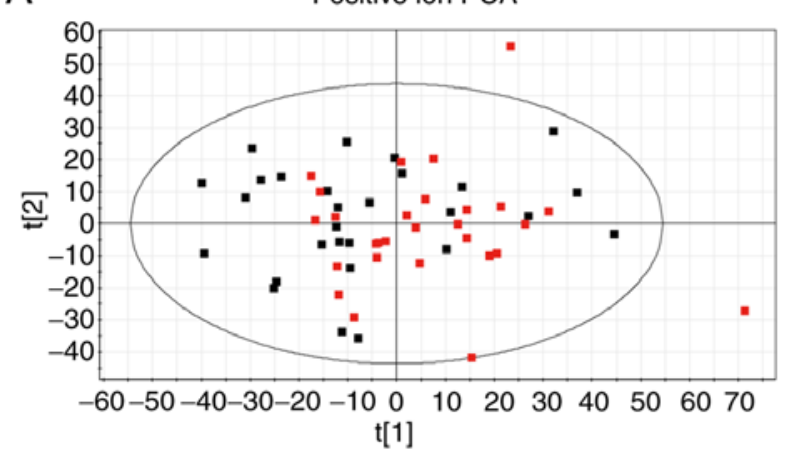

C

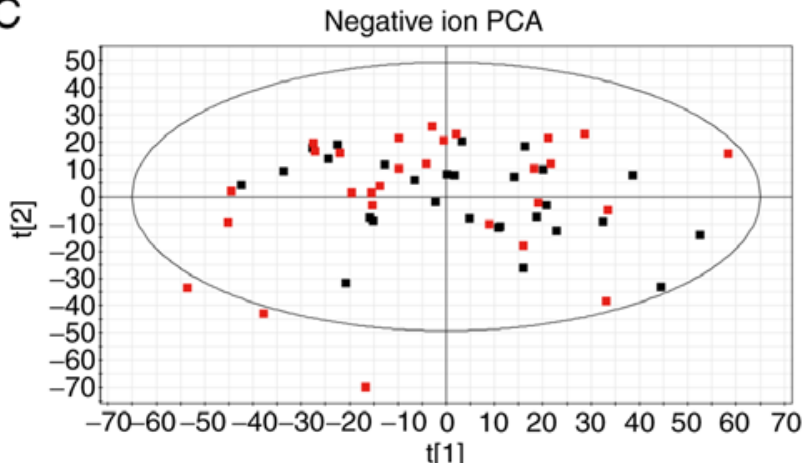

B
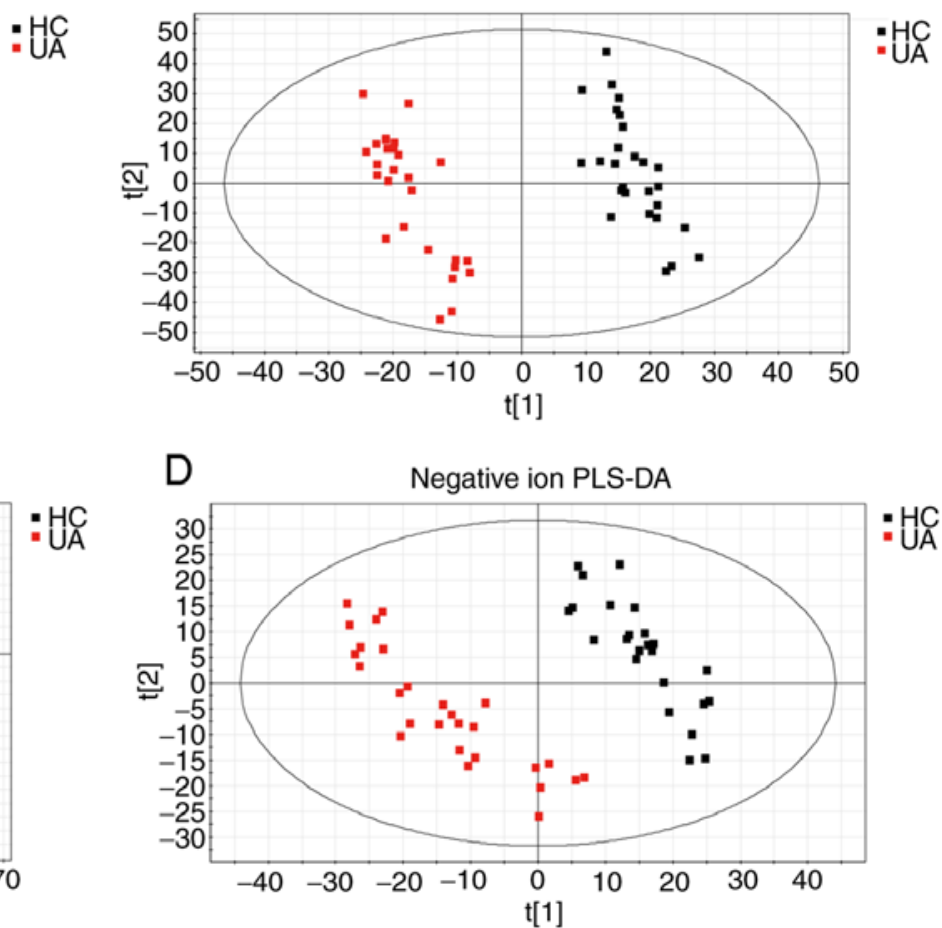

Figure 2. PCA and PLS-DA multivariate statistical analysis for UA and HC groups. (A) PCA and (B) PLS-DA scores of UA and HCs in positive ion mode. (C) PCA and (D) PLS-DA scores of UA and HCs in negative ion mode. PCA, principal component analysis; PLS-DA, partial least squares discriminant analysis; UA, unstable angina; $\mathrm{HC}$, health control.

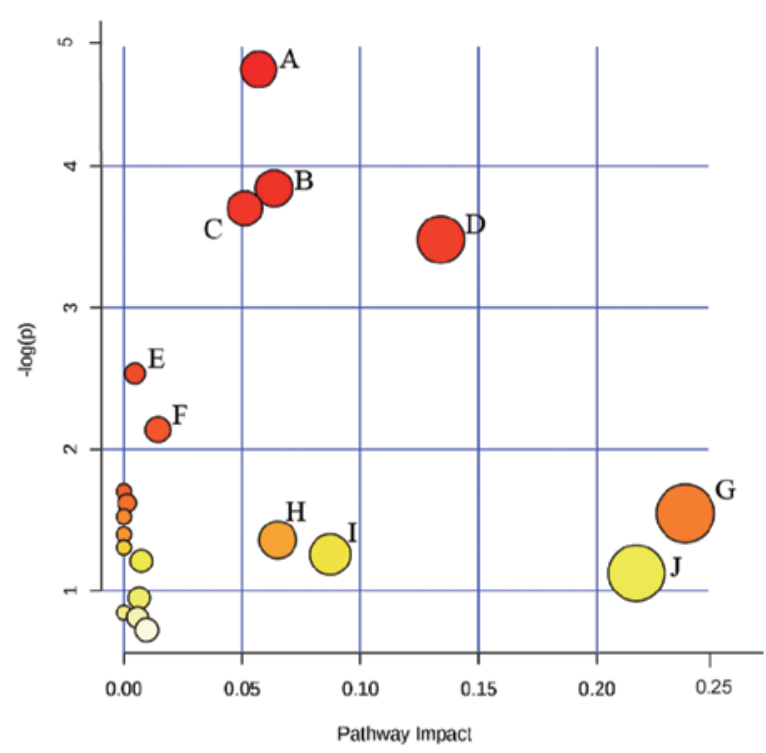

Figure 3. Summary of pathway analysis with MetPA for unstable angina. (A) 'Alanine, aspartate and glutamate metabolism' ( $\mathrm{P}=0.009$; impact $=0.057)$. (B) 'Steroid hormone biosynthesis' ( $\mathrm{P}=0.021$; impact=0.063). (C) 'Butanoate metabolism', ( $\mathrm{P}=0.025$; impact=0.051). (D) 'Ascorbate and aldarate metabolism'. (E) 'Tyrosine metabolism'. (F) 'Citrate cycle'. (G) 'Glutathione metabolism'. (H) 'Lysine degradation'. (I) 'Pentose and glucuronate interconversions'. (J) 'Arachidonic acid metabolism'. The impact value is proportional to the size of the circle. P-values are represented by a colour scale from yellow (low P-value) to red (high P-value).

ROC curve analysis of potential biomarkers. After 16 potential UA biomarkers were identified, ROC curves were constructed and used to determine whether these biomarkers exhibited diagnostic significance for UA. The different colored ROC curves in Fig. 4A represent an individual diagnostic biomarker. The AUC for each diagnostic biomarker was $>0.6$ (Table IV). Overall, the 16 potential biomarkers demonstrated diagnostic value for UA in Fig. 4B. The AUC for all diagnostic biomarkers was 1 , indicating a good accuracy for UA diagnosis.

Correlation analysis of potential biomarkers. The correlation between the 16 UA biomarkers was subsequently determined (Fig. 5). The results revealed that D-glucuronic acid was strongly correlated with creatinine, succinic acid and $\mathrm{N}$-acetylneuraminic acid, (Pearson's r, 0.449, 0.527 and 0.502, respectively; $\mathrm{P}<0.001$ ), suggesting that UA may be caused by a metabolic disorder. The correlation coefficient between adenine and arachidonic acid was $0.488(\mathrm{P}<0.001)$, indicating a potential role for purine and fatty acid metabolism in UA. In addition, the correlation coefficient between 2-octenedioic acid and sebacic acid was $0.669(\mathrm{P}<0.001)$, suggesting a potential association between fatty acid metabolism and UA. Other biomarkers were also closely correlated, such as porphobilinogen, cortisol and glutathione, which may explain the pathogenesis of UA.

\section{Discussion}

The current study identified 16 UA biomarkers via urinary metabolomics analysis. The associations among these biomarkers identified metabolic pathways that may be involved in UA. Amino acid and energy metabolism, fatty acid metabolism, purine metabolism and steroid hormone 
Table IV. AUC of metabolites associated with unstable angina.

Asymptotic 95\% CI

\begin{tabular}{lcccc} 
Compound & AUC & Standard error & Lower bound & Upper bound \\
\hline Sebacic acid & 0.714 & 0.068 & 0.581 & 0.848 \\
Succinic acid & 0.778 & 0.063 & 0.655 & 0.901 \\
Creatinine & 0.670 & 0.072 & 0.528 & 0.812 \\
Glutaric acid & 0.810 & 0.057 & 0.699 & 0.921 \\
Adenine & 0.656 & 0.073 & 0.512 & 0.800 \\
D-Glucurono-6,3-lactone & 0.618 & 0.076 & 0.468 & 0.768 \\
D-Glucuronic acid & 0.697 & 0.071 & 0.557 & 0.837 \\
N-Acetylneuraminic acid & 0.753 & 0.066 & 0.624 & 0.881 \\
2-Octenedioic acid & 0.682 & 0.071 & 0.542 & 0.822 \\
Estradiol & 0.686 & 0.072 & 0.545 & 0.827 \\
Porphobilinogen & 0.716 & 0.071 & 0.576 & 0.855 \\
Dihydrotestosterone & 0.693 & 0.073 & 0.550 & 0.835 \\
Glutathione & 0.760 & 0.064 & 0.635 & 0.885 \\
Succinic acid semialdehyde & 0.755 & 0.066 & 0.625 & 0.885 \\
Arachidonic acid & 0.675 & 0.075 & 0.529 & 0.822 \\
Cortisol & 0.724 & 0.067 & 0.593 & 0.856 \\
\hline
\end{tabular}

AUC, are under the curve.

A

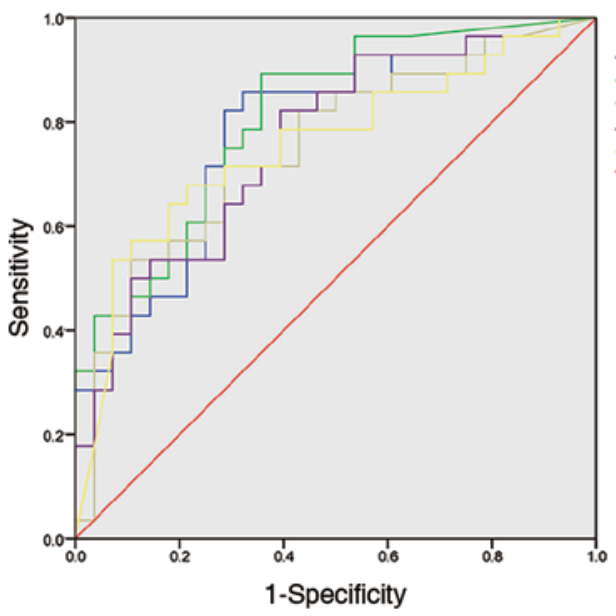

B

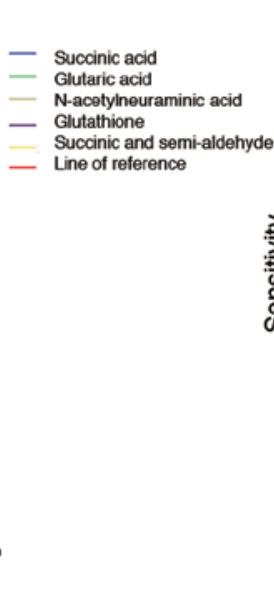

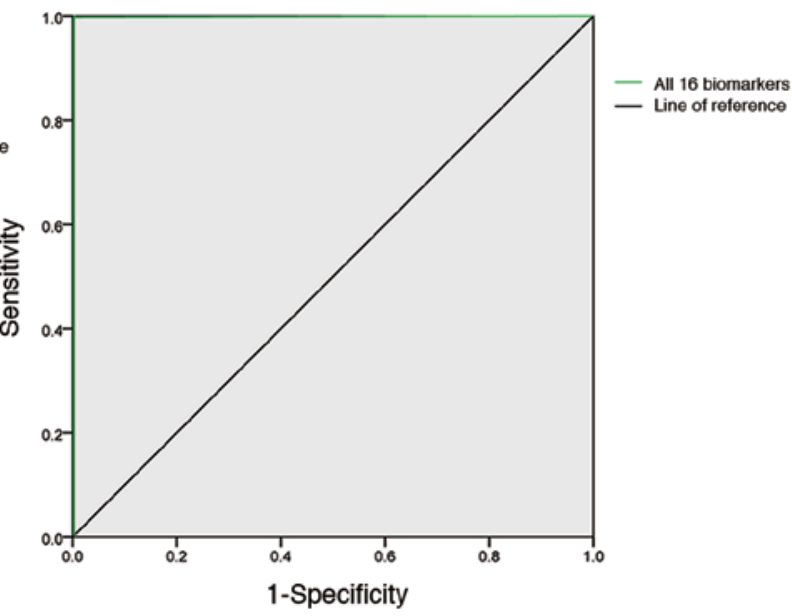

Figure 4. ROC curves of metabolites associated with unstable angina. (A) Single ROC curve of 16 potential biomarkers. (B) Overall ROC curve of 16 potential biomarkers. ROC, receiver operating characteristic.

biosynthesis metabolic pathways may be the main underlying cause of UA. The biomarkers and associated-metabolic pathways are presented in Fig. 6.

Amino acid metabolism, as a precursor of energy metabolism, serves a crucial role in increasing the formation of ATP (25). Glucuronolactone is a stable form of glucuronic acid; since glucuronic acid is usually absent in free fatty acids, an increase of glucuronic acid and a decrease of glucuronolactone indicates that amino acid metabolism may be disturbed in patients with UA (26). Creatinine arises from creatine and phosphocreatine, which is a type of nitrogenous organic acid naturally produced in the human body (27-29). In normal biological systems, the majority of creatine is converted to phosphocreatine by reversible enzymes, increasing creatine phosphate levels and maintaining and improving ATP levels during tissue oxygen depletion $(30,31)$. In the absence of enzymes, creatine is converted to creatinine $(32,33)$. A previous study analyzed the plasma creatinine level of 10,489 individuals and found that with the increase of age and creatinine level, the cumulative incidence of myocardial infarction and ischemic heart disease increased and the survival rate decreased (logarithmic rank trend $<0.001$ ), suggesting that creatinine is related to heart disease (34). 


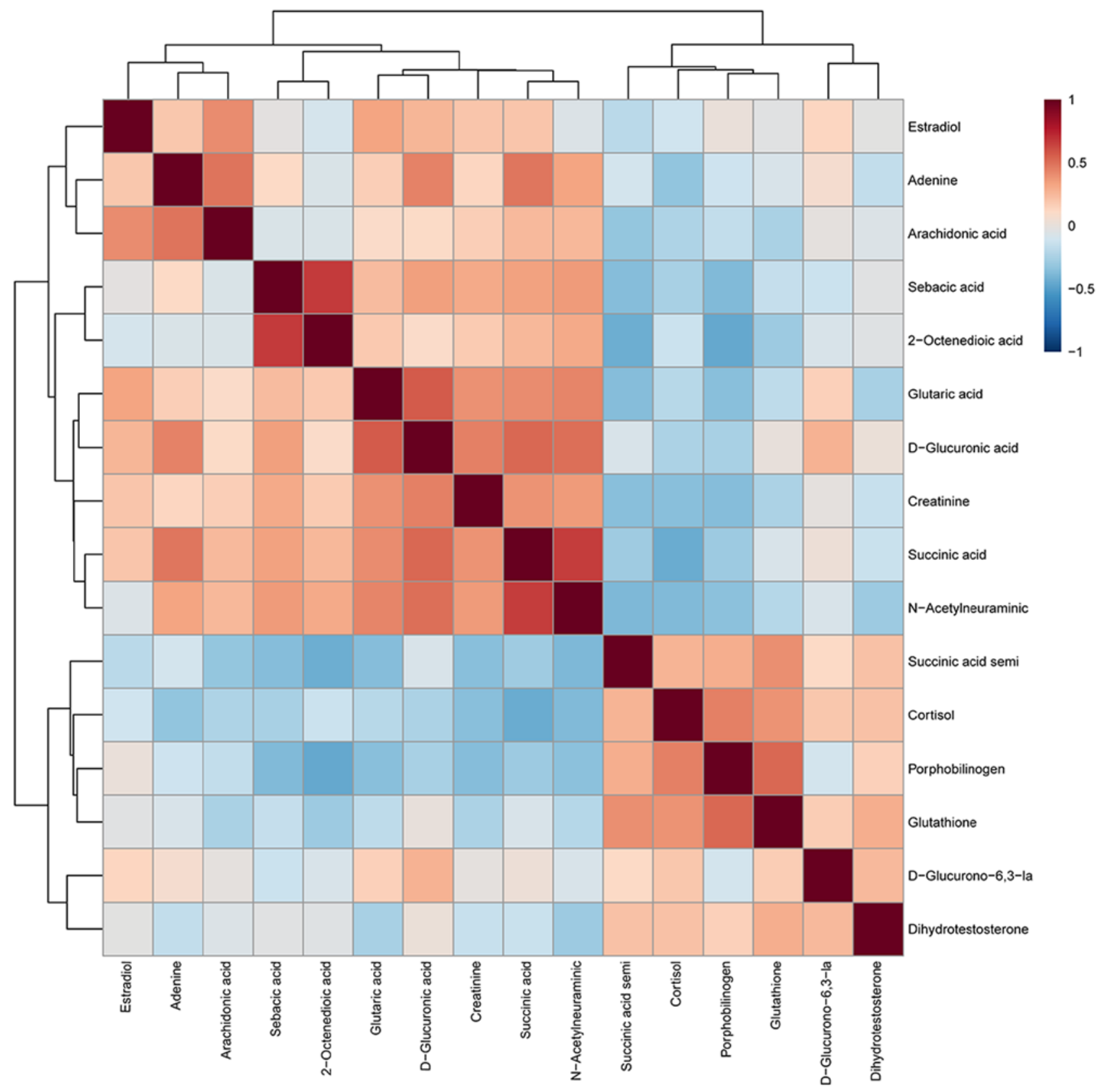

Figure 5. Correlation analysis of biomarkers in unstable angina. The different colors in the heatmap represent the different correlation coefficients between biomarkers; the darker the color of the block indicates higher correlation coefficients.

Glutathione is an endogenous antioxidant that protects cells from reactive oxygen species, which makes it essential for maintaining an appropriate immune response (35). A previous study has demonstrated that glutathione peroxidase deficiency overstimulates the proinflammatory pathway, leading to endothelial dysfunction and the development of cardiovascular disease (36). Additionally, the low glutathione peroxidase is associated with UA severity $(37,38)$. Succinic acid hemialdehyde can be oxidized by succinic acid dehydrogenase to succinic acid, which is an intermediate in the TCA cycle (39). A previous study has demonstrated that the accumulation of succinic acid in tissue is a universal metabolic feature of ischemia (39). The secretion of succinic acid induces inflammation and neovascularization, which may contribute to the pathogenesis of cardiovascular disease (40).
$\mathrm{N}$-acetylneuraminic acid is an $\mathrm{N}$-acetylated derivative of neuraminic acid, which is regulated by neuraminidase-1 (41). Zhang et al (41) used functional metabolomics to explore its role as a signaling molecule in triggering myocardial damage, and identified a key role for $\mathrm{N}$-acetylneuraminic acid in acute myocardial infarction, and targeting neuraminidase-1 may represent an unrecognized therapeutic intervention for CAD coronary artery disease.

Fatty acids are the main source of energy for the heart, accounting for $40-80 \%$ of the heart's energy source (21). A previous study has demonstrated that elevated free fatty acids levels are simply a biomarker of poor overall health, associated with an increased risk of cardiovascular (42). Glutaric, octenedioic and sebacic acids are dicarboxylic acids with 5 , 8 and 10 carbon atoms, respectively; they are converted into 


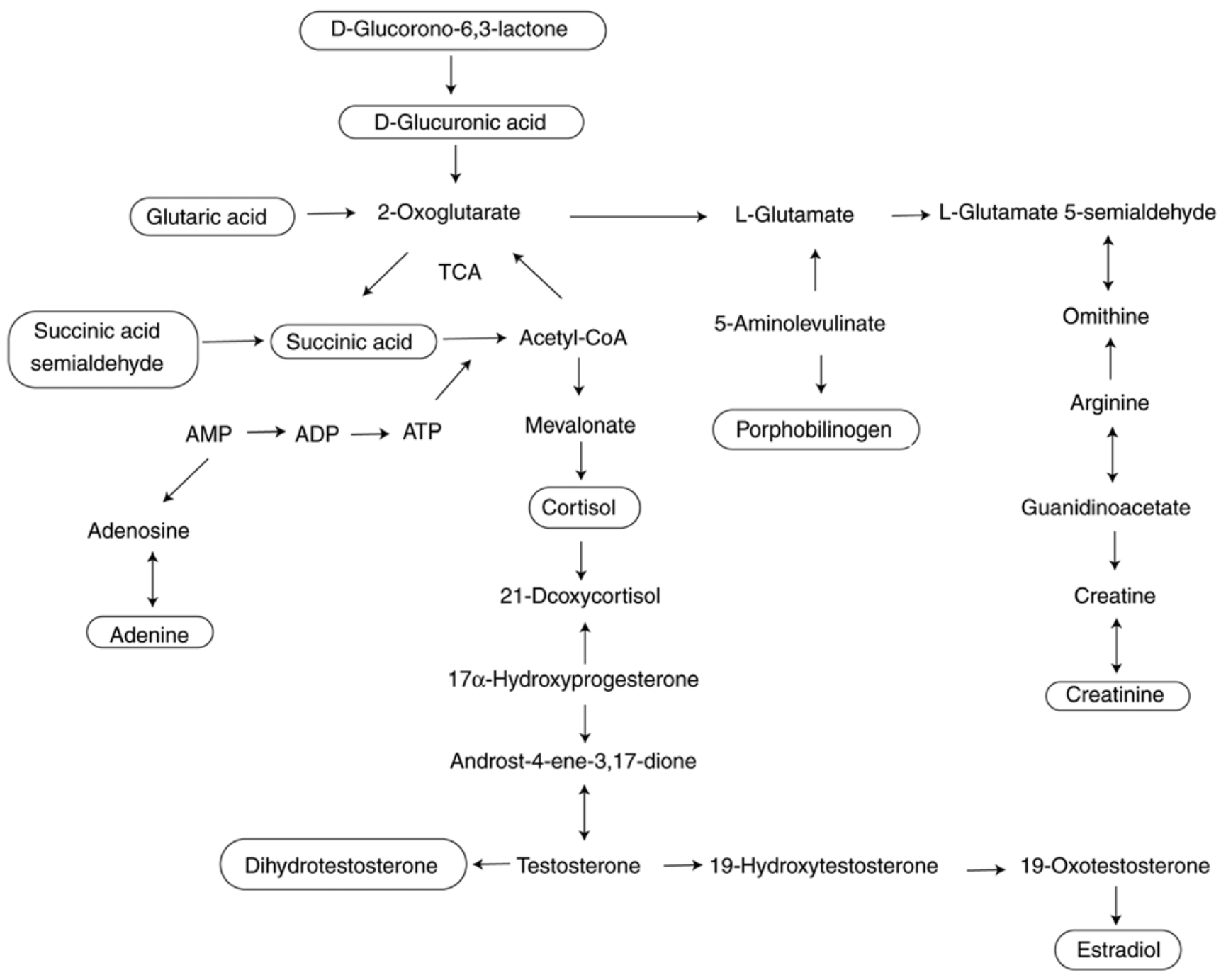

Figure 6. Metabolites and their corresponding pathways in the urine of patients with unstable angina. TCA, tricarboxylic acid.

acetyl-CoA through oxidation, which is subsequently further oxidized into the TCA cycle, providing necessary energy for the body $(43,44)$. Compared with the healthy controls, these biomarkers are significantly increased in the urine of patients with UA, indicating that peroxisome dysfunction and $\beta$-oxidation processes are reduced, causing excessive accumulation of dicarboxylic acid, it causes swelling of mitochondria and inhibits respiration of mitochondria $(45,46)$. It has also been reported that high levels of glutaric acid indicates that the body may be more susceptible to hematological toxicity (47). Arachidonic acid is a polyunsaturated $\omega-6$ fatty acid that occurs in the phospholipids of cell membranes; it is a precursor molecule during the synthesis of thromboxane A2 (48). Arachidonic acid generates thromboxane A2 through the thromboxane pathway to cause platelet aggregation, which triggers various cardiovascular diseases (49). For instance, previous study has shown that platelet aggregation induced by thromboxane A2 produced by arachidonic acid metabolism and thrombosis mediated by vasoconstriction sometimes cause myocardial infarction (50). Other studies have also shown that thromboxane A2, isoprostaglandin and prostacyclin can promote the formation and development of atherosclerosis by controlling platelet activation and leukocyte-endothelial cell interaction $(51,52)$. Platelet activation serves a role in blood clotting and thrombosis, and high levels of arachidonic acid are therefore associated with cardiovascular disease (53).

Platelet activation serves an important role in the pathology of UA (54). Under normal circumstances, uric acid is in a state of metabolic equilibrium. Elevated levels of adenine can cause an imbalance of uric acid metabolism, further promoting platelet aggregation (55). Highuric acid levels are associated with the mortality of patients with cardiovascular disease $(56,57)$. The antioxidant function of uric acid was previously considered to result in resistance to aging and oxidative stress; however, a recent clinical study has demonstrated that hyperuricemia may be one of the risk factors for cardiovascular disease (58).

Endogenous androgens represent a cardiovascular risk factor in humans (59). Previous studies have demonstrated that low testosterone levels can lead to an increased risk of cardiovascular disease (60-62). Dihydrotestosterone is an important hemostatic steroid with inhibitory effects on platelets (63). Dihydrotestosterone cannot be converted to estrogen by aromatase, but a previous study has indicated that testosterone can be converted to estradiol and act through estrogen receptors (64). In the current study, a significant decrease in dihydrotestosterone and estradiol levels were 
observed in patients with UA, indicating that the body had entered extreme androgen deficiency, which may lead to cardiovascular disease. In addition, high levels of cortisol may lead to increased cardiovascular disease risk through its associated effects on glucose, blood pressure and lipid metabolic functions (65). Porphobilinogen can generate heme via porphobilinogen deaminase; a previous study has demonstrated that angioedema may be accompanied with the temporary destruction of the blood-brain barrier, which may be induced by intermediates in the heme biosynthetic pathway (66).

Metabolomics systematically reveals the molecular mechanism of metabolic pathways, determining the pathophysiological processes of disease development and searching for novel disease biomarkers. Based on the established non-targeted metabolomics analysis platform, the current study assessed 16 biomarkers that were associated with UA by analyzing the levels of metabolites in patient urine. The results revealed strong correlations between 2-octenedioic acid and sebacic acid, amino acid metabolism, fatty acid metabolism, purine metabolism and biosynthesis and steroid hormone metabolism, indicating that these metabolic pathways were affected in patients with UA pectoris. These pathways may also be associated with UA pathogenesis. The results of the present study also demonstrated the potential of biomarkers to assist in the early diagnosis of UA, thus providing patients with personalized clinical solutions that enable effective treatment of UA within a feasible time frame, preventing progression to acute myocardial ischemia and myocardial infarction. However, the biological mechanisms of abnormal metabolic biomarkers in metabolic pathways requires further study to provide more information for the clinical diagnosis and treatment of UA.

\section{Acknowledgements}

Not applicable.

\section{Funding}

The present study was supported by the National Key Basic Research and Development Program (973 Program; grant no. 2014CB542902).

\section{Availability of data and materials}

The datasets used and/or analyzed during the present study are available from the corresponding author on reasonable request.

\section{Authors' contributions}

YBL and CY researched the literature and conceived the current study. SG and SF developed the protocol and obtained the ethics approval. XC, YJL and ZL recruited the participants and performed the statistical analysis. $\mathrm{HZ}$ and $\mathrm{TZ}$ analyzed the data. YCL and YL performed the experiments and wrote the first draft of the manuscript. All authors reviewed and edited the manuscript. All authors read and approved the final manuscript.

\section{Ethics approval and consent to participate}

The trial was registered with the Chinese Clinical Trial Registration Center (registration no. ChiCTR-ROC-17013957) at the Tianjin University of Traditional Chinese Medicine. The current study conforms to the Declaration of Helsinki and was approved by the Ethics Committee of Tianjin University of Traditional Chinese Medicine (approval no. TJUTCM-EC20180003).

\section{Patient consent for publication}

Not applicable.

\section{Competing interests}

The authors declare that they have no competing interests.

\section{References}

1. Benjamin EJ, Virani SS, Callaway CW, Chamberlain AM, Chang AR, Cheng S, Chiuve SE, Cushman M, Delling FN, Deo R, et al: Heart disease and stroke statistics-2018 update: A report from the American heart association. Circulation 137: e67-e492, 2018.

2. D'Souza M, Sarkisian L, Saaby L, Poulsen TS, Gerke O, Larsen TB, Diederichsen AC, Jangaard N, Diederichsen SZ, Hosbond S, et al: Diagnosis of unstable angina pectoris has declined markedly with the advent of more sensitive troponin assays. Am J Med 128: 852-860, 2015.

3. Möckel M, Searle J, Hamm C, Slagman A, Blankenberg S, Huber K, Katus H, Liebetrau C, Müller C, Muller R, et al: Early discharge using single cardiac troponin and copeptin testing in patients with suspected acute coronary syndrome (ACS): A randomized, controlled clinical process study. Eur Heart J 36: 369-376, 2015.

4. Amandeep G and Roman Z: Unstable Angina. Treasure Island (FL): StatPearls Publishing, 2020.

5. Yeghiazarians Y, Braunstein JB, Askari A and Stone PH: Unstable angina pectoris. New Engl J Med 342: 101-114, 2000.

6. Sun M, Gao X, Zhang D, Ke C, Hou Y, Fan L, Zhang R, Liu H, $\mathrm{Li} \mathrm{K}$ and $\mathrm{Yu} \mathrm{B}$ : Identification of biomarkers for unstable angina by plasma metabolomic profiling. Mol Biosyst 9: 3059-3067, 2013.

7. Anderson JL, Horne BD, Stevens SM, Grove AS, Barton S, Nicholas ZP, Kahn SF, May HT, Samuelson KM, Muhlestein JB, et al: Randomized trial of genotype-guided versus standard warfarin dosing in patients initiating oral anticoagulation. Circulation 116: 2563-2570, 2007.

8. David SW: Metabolomics for investigating physiological and pathophysiological processes. Physiol Rev 99: 1819-1875, 2019.

9. Cheung PK, Ma MH, Tse HF, Yeung KF, Tsang HF, Chu MKM, Kan CM, Cho WCS, Ng LBW, Chan LWC and Wong SCC: The applications of metabolomics in the molecular diagnostics of cancer. Expert Rev Mol Diagn 19: 785-793, 2019.

10. Wheelock CE, Wheelock AM, Kawashima S, Diez D, Kanehisa M, van Erk M, Kleemann R, Haeggström JZ and Goto S: Systems biology approaches and pathway tools for investigating cardiovascular disease. Mol Biosyst 5: 588-602, 2009.

11. Patti GJ, Yanes O and Siuzdak G: Innovation: Metabolomics: The apogee of the omics trilogy. Nat Rev Mol Cell Biol 13: 263-269, 2012

12. Long NP, Nghi TD, Kang YP, Anh NH, Kim HM, Park SK and Kwon SW: Toward a standardized srategy of clinical metabolomics for the advancement of precision medicine. Metabolites 10: $51,2020$.

13. Nicholson JK and Lindon JC: Systems biology: Metabonomics. Nature 455: 1054-1056, 2008.

14. Strimbu K and Tavel JA: What are biomarkers? Curr Opin HIV AIDS 5: 463-466, 2010.

15. Bujak R, Struck-Lewicka W, Markuszewski MJ and Kaliszan R: Metabolomics for laboratory diagnostics. J Pharm Biomed Anal 113: 108-120, 2015. 
16. González-Domínguez R, García-Barrera T and Gómez-Ariza JL: Combination of metabolomic and phospholipid-profiling approaches for the study of Alzheimer's disease. J Proteomics 104 37-47, 2014.

17. Psychogios N, Hau DD, Peng J, Guo AC, Mandal R, Bouatra S, Sinelnikov I, Krishnamurthy R, Eisner R, Gautam B, et al: The human serum metabolome. PLoS One 6: e16957, 2011.

18. Bouatra S, Aziat F, Mandal R, Guo AC, Wilson MR, Knox C, Bjorndahl TC, Krishnamurthy R, Saleem F, Liu P, et al: The human urine metabolome. PLoS One 8: e73076, 2013.

19. Zhang M, Fu G and Lei T: Two Urinary Peptides Associated Closely With Type 2 Diabetes Mellitus. PLoS One 10: e0122950, 2015

20. Li XS, Li S and Kellermann G: Pre-analytical and analytical validations and clinical applications of a miniaturized, simple and cost-effective solid phase extraction combined with LC-MS/MS for the simultaneous determination of catecholamines and metanephrines in spot urine samples. Talanta 159: 238-247, 2016.

21. Wang Y, Sun W, Zheng J, Xu C, Wang X, Li T, Tang Y and Li Z: Urinary metabonomic study of patients with acute coronary syndrome using UPLC-QTOF/MS. J Chromatogr B Analyt Technol Biomed Life Sci 1100: 122-130, 2018.

22. Li J, Guo J, Shang E, Zhu Z, Zhu KY, Li S, Zhao B, Jia L, Zhao J, et al: A metabolomics strategy to explore urinary biomarkers and metabolic pathways for assessment of interaction between Danhong injection and low-dose aspirin during their synergistic treatment. J Chromatogr B Analyt Technol Biomed Life Sci 1026: 168-175, 2016

23. Kang SM, Park JC, Shin MJ, Lee H, Oh J, Ryu DH, Hwang GS and Chung JH: ${ }^{1} \mathrm{H}$ nuclear magnetic resonance based metabolic urinary profiling of patients with ischemic heart failure. Clin Biochem 44: 293-299, 2011

24. Chinese Medical Association Cardiovascular Branch, Editorial Board of the Chinese Journal of Cardiovascular Diseases: Guidelines for the diagnosis and treatment of unstable angina and non-ST-segment elevation myocardial infarction. Chin J Cardiol 35: 25-304, 2007.

25. Guo N, Yang D, Wang X, Dai J, Wang M and Lei Y: Metabonomic study of chronic heart failure and effects of Chinese herbal decoction in rats. J Chromatogr A 1362: 89-101, 2014.

26. Bai J, Wang MX, Chowbay B, Ching CB and Chen WN: Metabolic profiling of HepG2 cells incubated with $\mathrm{S}(-)$ and $\mathrm{R}(+)$ enantiomers of anti-coagulating drug warfarin. Metabolomics 7: 353-362, 2011

27. Feng Q, Liu Z, Zhong S, Li R, Xia H, Jie Z, Wen B, Chen X, Yan W, Fan Y, et al: Integrated metabolomics and metagenomics analysis of plasma and urine identified microbial metabolites associated with coronary heart disease. Sci Rep 6: 22525, 2016.

28. Wyss $M$ and Kaddurah-Daouk R: Creatine and creatinine metabolism. Physiol Rev 80: 1107-1213, 2000.

29. Zheng P, Gao HC, Li Q, Shao WH, Zhang ML, Cheng K, Yang DY, Fan SH, Chen L and Xie P: Plasma metabonomics as a novel diagnostic approach for major depressive disorder. J Proteome Res 11: 1741-1748, 2012.

30. Chen F, Zhu K, Chen L, Ouyang L, Chen C, Gu L, Jiang Y, Wang Z, Lin Z, Zhang Q, et al: Protein target identification of ginsenosides in skeletal muscle tissues: Discovery of natural small-molecule activators of muscle-type creatine kinase. J Ginseng Res 44: 461-474, 2020.

31. Kallioniemi E, Kärkkäinen $O$, Määttä S, Könönen $M$, Kivimäki P, Kaarre O, Velagapudi V, Kekkonen V, Lehto SM, Laukkanen E and Tolmunen T: Repeated transcranial magnetic stimulation-induced motor evoked potentials correlate with the subject-specific serum metabolic profile of creatine. J Clin Neurophysiol 36: 229-235, 2019.

32. Dickinson H, Ellery S, Ireland Z, LaRosa D, Snow R and Walker DW: Creatine supplementation during pregnancy: Summary of experimental studies suggesting a treatment to improve fetal and neonatal morbidity and reduce mortality in high-risk human pregnancy. BMC Pregnancy Childbirth 14: 150, 2014.

33. Li Z, Liu X, Wang J, Gao J, Guo S, Gao K, Man H, Wang Y, Chen $\mathrm{J}$ and Wang $\mathrm{W}$ : Analysis of urinary metabolomic profiling for unstable angina pectoris disease based on nuclear magnetic resonance spectroscopy. Mol Biosyst 11: 3387-3396, 2015.

34. Sibilitz KL, Benn M and Nordestgaard BG: Creatinine, eGFR and association with myocardial infarction, ischemic heart disease and early death in the general population. Atherosclerosis 237: $67-75,2014$
35. Mourino-Alvarez L, Baldan-Martin M, Gonzalez-Calero L, Martinez-Laborde C, Sastre-Oliva T, Moreno-Luna R, Lopez-Almodovar LF, Sanchez PL, Fernandez-Aviles F, Vivanco F, et al: Patients with calcific aortic stenosis exhibit systemic molecular evidence of ischemia, enhanced coagulation, oxidative stress and impaired cholesterol transport. Int J Cardiol 225: 99-106, 2016.

36. Sharma A, Yuen D, Huet O, Pickering R, Stefanovic N, Bernatchez $\mathrm{P}$ and de Haan JB: Lack of glutathione peroxidase-1 facilitates a pro-inflammatory and activated vascular endothelium. Vascul Pharmacol 79: 32-42, 2016.

37. Rosenblat M, Volkova N, Coleman R and Aviram M: Anti-oxidant and anti-atherogenic properties of liposomal glutathione: Studies in vitro, and in the atherosclerotic apolipoprotein E-deficient mice. Atherosclerosis 195: e61-e68, 2007.

38. Qiao M, Kisgati M, Cholewa JM, Zhu W, Smart EJ, Sulistio MS and Asmis R: Increased expression of glutathione reductase in macrophages decreases atherosclerotic lesion formation in low-density lipoprotein receptor-deficient mice. Arterioscler Thromb Vasc Biol 27: 1375-1382, 2007.

39. Chouchani ET, Pell VR, Gaude E, Aksentijević D, Sundier SY, Robb EL, Logan A, Nadtochiy SM, Ord ENJ, Smith AC, et al: Ischaemic accumulation of succinate controls reperfusion injury through mitochondrial ROS. Nature 515: 431-435, 2014.

40. Hamel D, Sanchez M, Duhamel F, Roy O, Honoré JC, Noueihed B, Zhou T, Nadeau-Vallée M, Hou X, Lavoie JC, et al: G-protein-coupled receptor 91 and succinate are key contributors in neonatal postcerebral hypoxia-ischemia recovery. Arterioscler Thromb Vasc Biol 34: 285-293, 2014.

41. Zhang L, Wei TT, Li Y, Li J, Fan Y, Huang FQ, Cai YY, Ma GX, Liu JF, et al: Functional metabolomics characterizes a key role for n-acetylneuraminic acid in coronary artery diseases. Circulation 137: 1374-1390, 2018.

42. Miedema MD, Maziarz M, Biggs ML, Zieman SJ, Kizer JR, Ix JH, Mozaffarian D, Tracy RP, Psaty BM, Siscovick DS, et al: Plasma-free fatty acids, fatty acid-binding protein 4 , and mortality in older adults (from the Cardiovascular Health Study). Am J Cardiol 114: 843-848, 2014.

43. Mingrone G, Castagneto-Gissey L and Macé K: Use of dicarboxylic acids in type 2 diabetes. Br J Clin Pharmacol 75: 671-676, 2013.

44. Jia P, Wang S, Xiao C, Yang L, Chen Y, Jiang W, Zheng X, Zhao G, Zang W and Zheng X: The anti-atherosclerotic effect of tanshinol borneol ester using fecal metabolomics based on liquid chromatography-mass spectrometry. Analyst 141: 1112-1120, 2016.

45. Nazzaro-Porro M: Azelaic acid. J Am Acad Dermatol 17: 1033-1041, 1987.

46. Wang SY, Wang Y, Jin XW, Zhang Y, Chen JS, Ma WW, Wu YH and Wang DC: A urinary metabolomics study of rats after the exposure to acrylamide by ultra performance liquid chromatography coupled with quadrupole time-of-flight tandem mass spectrometry. Mol Biosyst 11: 1146-1155, 2015.

47. Nishiumi S, Fujigaki S, Kobayashi T, Kojima T, Ito Y, Daiko H, Kato K, Shoji H, Kodama Y, Honda K and Yoshida M: Metabolomics-based discovery of serum biomarkers to predict the side-effects of neoadjuvant chemoradiotherapy for esophageal squamous cell carcinoma. Anticancer Res 39: 519-526, 2019.

48. Patrono C and Rocca B: Measurement of thromboxane biosynthesis in health and disease. Front Pharmacol 10: 1244, 2019.

49. Hirz T, Khalaf A, El-Hachem N, Mrad MF, Abdallah H, Créminon C, Grée R, Merhi RA, Habib A, Hachem A and Hamade E: New analogues of 13-hydroxyocatdecadienoic acid and 12-hydroxyeicosatetraenoic acid block human blood platelet aggregation and cyclooxygenase-1 activity. Chem Cent J 6: 152, 2012.

50. Nielsen MS, Schmidt EB, Stegger J, Gorst-Rasmussen A, Tjonneland A and Overvad K: Adipose tissue arachidonic acid content is associated with the risk of myocardial infarction: A Danish case-cohort study. Atherosclerosis 227: 386-390, 2013.

51. Smyth EM: Thromboxane and the thromboxane receptor in cardiovascular disease. Clin Lipidol 5: 209-219, 2010.

52. Martin W: The combined role of atheroma, cholesterol, platelets, the endothelium and fibrin in heart attacks and strokes. Med Hypotheses 15: 305-322, 1984.

53. Jarrar YB, Cho SA, Oh KS, Kim DH, Shin JG and Lee SJ: Identification of cytochrome P450s involved in the metabolism of arachidonic acid in human platelets. Prostaglandins Leukot Essent Fatty Acids 89: 227-234, 2013. 
54. Ameta K, Gupta A, Ameta D, Sethi R, Kumar D, Ahmad I and Mahdi AA: 1H NMR-derived metabolomics of filtered serum of myocardial ischemia in unstable angina patients. Clin Chim Acta 456: 56-62, 2016.

55. Boyle SH, Matson WR, Velazquez EJ, Samad Z, Williams RB Jr, Sharma S, Thomas B, Wilson JL, O'Connor C and Jiang W: Metabolomics analysis reveals insights into biochemical mechanisms of mental stress-induced left ventricular dysfunction. Metabolomics 11: 571-582, 2015.

56. Krishnan E, Kwoh CK, Schumacher HR and Kuller L: Hyperuricemia and incidence of hypertension among men without metabolic syndrome. Hypertension 49: 298-303, 2007.

57. Baker JF, Krishnan E, Chen L and Schumacher HR: Serum uric acid and cardiovascular disease: Recent developments, and where do they leave us? Am J Med 118: 816-826, 2005.

58. Lippi G, Montagnana M, Franchini M, Favaloro EJ and Targher G: The paradoxical relationship between serum uric acid and cardiovascular disease. Clin Chim Acta 392: 1-7, 2008.

59. Ajayi AA, Mathur R and Halushka PV: Testosterone increases human platelet thromboxane A2 receptor density and aggregation responses. Circulation 91: 2742-2747, 1995.

60. Karolczak K, Konieczna L, Kostka T, Witas PJ, Soltysik B, Baczek $\mathrm{T}$ and Watala C: Testosterone and dihydrotestosterone reduce platelet activation and reactivity in older men and women. Aging (Albany NY) 10: 902-929, 2018.

61. Shiraki N, Nakashima A, Doi S, Carrero JJ, Sugiya N, Ueno T, Stenvinkel P, Kohno N and Masaki T: Low serum testosterone is associated with atherosclerosis in postmenopausal women undergoing hemodialysis. Clin Exp Nephrol 18: 499-506, 2014
62. Shores MM and Matsumoto AM: Testosterone, aging and survival: Biomarker or deficiency. Curr Opin Endocrinol Diabetes Obes 21: 209-216, 2014.

63. Chasland LC, Knuiman MW, Divitini ML, Murray K, Handelsman DJ, Flicker L, Hankey GJ, Almeida OP, Golledge J, Ridgers ND, et al: Higher circulating androgens and higher physical activity levels are associated with less central adiposity and lower risk of cardiovascular death in older men. Clin Endocrinol (Oxf) 90: 375-383, 2019.

64. Rosenberg MA, Shores MM, Matsumoto AM, Bůžková P, Lange LA, Kronmal RA, Heckbert SR and Mukamal KJ: Serum androgens and risk of atrial fibrillation in older men: The cardiovascular health study. Clin Cardiol 41: 830-836, 2018

65. Lattanzi S and Silvestrini M: Letter re: Long-term cortisol measures predict Alzheimer disease risk. Neurology 89: 106 2017.

66. Maramattom BV, Zaldivar RA, Glynn SM, Eggers SD and Wijdicks EF: Acute intermittent porphyria presenting as a diffuse encephalopathy. Ann Neurol 57: 581-584, 2005.

This work is licensed under a Creative Commons Attribution-NonCommercial-NoDerivatives 4.0 International (CC BY-NC-ND 4.0) License. 